\title{
Estrategias para viabilizar la transferencia de conocimiento y tecnología generado en el Instituto de Investigación de la Facultad de Ciencias de la Administración de la UNCP, 2016
}

\author{
Strategies to enable knowledge transfer and technology generated at the \\ Research Institute of the Faculty of Administration Sciences of the UNCP, 2016
}

'Flores Gamboa, A.P.; 2 Samaniego Flores, C.P.

Facultad de Ciencias de la Administración, Universidad Nacional del Centro del Perú

Email: apflores@uncp.edu.pe

\section{Resumen}

La transferencia tecnológica es un tema preocupante a nivel nacional y el Concytec, con ayuda del Estado, instituciones académicas y empresariales, tiene el reto de afrontar este problema entre el año 2016 al 2021; ante esto, el Vicerrectorado de Investigación de la Universidad Nacional del Centro del Perú, está tomando medidas de mejora continua con respecto a la labor de investigación. Una de las estrategias principales es la transferencia tecnológica la cual permite conectar al investigador con la sociedad en un entorno empresarial.

La investigación tiene por objetivo identificar estrategias que permitan viabilizar la transferencia de conocimiento y tecnología en la Facultad de Ciencias de la Administración en bien de nuestra sociedad. Para lograr este objetivo, se identificó y clasificó los trabajos de investigación; de los 18 informes finales, del 2016 y 2017; el 61 \%, son investigaciones cuyos resultados resuelven problemas estudiantiles de Facultad de Ciencias de la Administración; $11 \%$, investigaciones realizadas en la UNCP; $17 \%$, son libros y; sólo el $11 \%$, son investigaciones fuera del ámbito de la Facultad.

Asimismo, se realizó una encuesta anónima referida a las dimensiones a considerar en la transferencia tecnológica a todos los 33 investigadores de la Facultad en el año 2016, de los cuales 25 son docentes y 8 administrativos. Se utilizó la prueba rho de Spearman, porque se quería demostrar si existe una relación entre las estrategias manejadas por el Instituto de investigacion que viabilicen a transferencia de conocimiento y tecnología.

Palabras Clave: transferencia de conocimientos, transferencia tecnológica, estrategias, Concytec, innovación tecnológica.

\begin{abstract}
The transfer of technology is a matter of national concern and the Concytec, with the help of the State, academic and business institutions have the challenge of facing this problem between 2016 to 2021, before this the Vice-Rector for Research of the National University of the Center of Peru is taking continuous improvement measures with respect to the research work. One of the main strategies is the transfer of technology which allows the researcher to connect with society in a business environment.
\end{abstract}

The objective of the research is to identify strategies that make viable the transfer of knowledge and technology in the Faculty of Administrative Sciences for the good of our society. To achieve this objective, the research work was identified and classified. Of the 18 final reports of 2016 and 2017, 61\% are Research whose results solve student problems in the Faculty of Administration Sciences, $11 \%$ research carried out in the UNCP, $17 \%$ are books, only $11 \%$ are research outside the scope of the Faculty.

Likewise, an anonymous survey was carried out regarding the dimensions to be considered in the technological transfer to all 33 researchers of the Faculty in 2016, of which 25 are teachers and 8 administrative. The Spearman rho test was used because it was wanted to demonstrate if there is a relationship between the strategies managed by the research institute that enable the transfer of knowledge and technology.

Keywords: Knowledge transfer, technology transfer, strategies, Concytec, technological innovation. 


\section{Introducción}

El presente trabajo de investigación surge por dos motivos muy importantes; la asistencia al diplomado de investigación, organizado por el Vicerrectorado de Investigación de la Universidad Nacional del Centro del Perú (UNCP) y; por otro lado, parte del consejo directivo de la Oficina General de Extensión Cultural Proyección Social y Transferencia Tecnológica (OGECPS y TT). En ambos eventos, uno de los temas principales fue la transferencia tecnológica. En estas circunstancias, la transferencia de conocimiento es un factor decisivo para ayudar al sector empresarial a lograr un aumento en el desarrollo de las innovaciones (Beraza \& Rodríguez, 2010).

Existe la preocupación en los docentes de la UNCP, por realizar trabajos de investigación por obtener una certificación para el nombramiento, ascenso y ratificación o poder ser considerado en la bonificación por investigación. Como resultado se presentan, trabajos sin trascendencia científica y que de ninguna manera lograrán la transferencia tecnológica y poder convertirse en artículos científicos que puedan publicarse en revistas indexadas, para así poder contribuir al desarrollo científico de la UNCP y de la sociedad. La explotación de los resultados de la investigación pública sigue siendo una asignatura pendiente para gran parte de los países. (Universitario \& Decisión, 2011)

El actual desarrollo de los países depende de su capacidad para generar, utilizar, transferir y difundir el conocimiento científico y tecnológico. En este marco, las universidades, como instituciones generadoras de conocimiento y formación, cumplen un rol protagónico en el desarrollo económico y social por medio de la transferencia de los resultados de investigación, ya sea como tecnología o know how y la formación de profesionales que respondan a las demandas y necesidades de la sociedad en su conjunto (Concytec, 2016); por consiguiente, si las investigaciones que realizan los docentes de Facultad de Ciencias de la Administración (FCA) responden a las inquietudes empresariales de nuestra región será más fácil de lograr la transferencia de conocimiento y tecnología y poder contar con artículos científicos en beneficio de la UNCP.

Algunos estudiosos destacan que las universidades y algunos centros de investigación, públicos o privados, son los principales impulsores de la generación y de la transferencia de conocimiento y de tecnología, lo que los ha llevado a tender puentes con los sectores productivos para dar respuesta, junto a aquello que demanda la sociedad (Vázquez, 2017). También, es importante mencionar el "modelo actual de actuación de las universidades, en concreto de su contribución a la innovación tecnológica” (Azagra, 2003).

En el año 2016, surgió la preocupación nacional del Concytec, sobre la trascendencia de la investigación científica, afirmando que no se está logrando una buena transferencia tecnológica, lo cual se evidencia en las actividades del sector productivo, basadas en la incorporación de tecnología empaquetada y no utilizando transferencia tecnológica. (Concytec, 2016).

En este contexto, la investigación analizó esta problemática en la FCA, evidenciándose lo siguiente: que los trabajos de investigación deben de salir del ámbito del programa o universidad y atender problemas reales de la sociedad, para poder facilitar la transferencia tecnológica y; los convenios que se realizan en los programas y centros de investigación deben ser en gran número con demandas específicas de las empresas y comunidades, los trabajos de investigación que se realizan son temas irrelevantes, que no se vinculan con el sector productivo e innovación tecnológica. Por lo expuesto, se formuló estrategias para viabilizar la transferencia de conocimiento y tecnología del conocimiento generado en el Instituto Especializado de Investigación de la Facultad de Ciencias de la Administración de la UNCP y el sector productivo tenga la debida confianza en las investigaciones que se realizan.

Según la información del director del Instituto Especializado de Investigación de la FCA, se cuenta con la participación de 25 docentes de diferentes categorías y 8 administrativos de diferentes oficinas administrativas de la UNCP, todos ellos nombrados. Con base en las informaciones del Instituto Especializado de Investigación de la FCA, el Concytec, ha planteado las estrategias que permita la transferencia de conocimientos y tecnología.

\section{Materiales y métodos}

El diseño de la investigación descriptiva- correlacional, la muestra estuvo conformada por todos los docentes investigadores de la Facultad de Ciencias de Administración de la Universidad Nacional del Centro del Perú en el año 2017, siendo un total de 33 investigadores, de los cuales 25 son docentes y 8 administrativos.

Se excluyó a los docentes y administrativos que no presentaron proyectos de investigación al Instituto Especializado de Investigación de la FCA. Se elaboró un cuestionario con 22 preguntas, fundamentados en la revisión bibliográfica y del análisis de algunos casos exitosos que potencialmente pueden incidir en el éxito de la investigación. (Barragán, 2010)

Los docentes y administrativos, fueron contactados por los investigadores y se obtuvieron los permisos verbales por ser miembros de la facultad.

Se utilizó la prueba rho de Spearman, porque se quiso demostrar si existe una relación entre las estrategias manejadas por el centro de investigación que viabilicen la transferencia de conocimiento y tecnología. 


\section{Resultados}

En la Tabla 1, se muestran los títulos de las 18 investigaciones inscritas en el Instituto Especializado de Investigación de la FCA, realizadas por docentes y administrativos en el año 2016.

Tabla 1

Títulos de las investigaciones del periodo 2017 - FCA UNCP

\section{Título}

1. Estudio de la demanda social y mercado ocupacional de la carrera profesıonal de admınıstración

2. Influencia de los usos de las TICs educatıvas en la satısfacción de los estudiantes en la Facultad de Ciencias de la Adminıstración.

3. La administración y los nuevos modelos de negocio.

4. Influencia del planeamıento estratégico en la ejecución presupuestal de la Unıversıdad Nacional del Centro del Perú

5. La calidad de servicio a los estudiantes de posgrado de la Facultad de Ciencias de la Adminıstración.

6. Factores que influyen en la competitıvidad de la carrera profesıonal de admınıstración de empresas de la UNCP en la región Junín 2016

7. Determınantes de la elección de una carrera unıversıtarıa en el Perú.

8. Problemarıo de matemátıca básıca

9. Medicıón de la calıdad de servicıo en la satısfacción de los usuarıos en el área adminıstratıva del Pabellón de Admınıstración y Gobıerno UNCP.

10. Perfil emprendedor de los estudiantes de la Facultad de Ciencias de la Admınıstración de la Unıversıdad Nacional del Centro del Perú - 2016

11. Factor "experiencia del postor" ¿habrá equilibrio combınación "número de veces valor referencial" y perıdo facturado? En licitaciones públicas - UNCP 2015

12. El clima organızacional y su influencia en el desempeño de los trabajadores de la Facultad de Ciencias de la Admınıstracıón de la Unıversıdad Nacıonal del Centro del Perú. 2016

13. La filosofía organızacional y la gestión de la Facultad de Ciencias de la Administración.

14. Metodología de la investıgacıón. Segunda edicıón.

15. Metodología de investıgación científica: guía de orıentación metodológica para la elaboración de proyectos e informes de investıgación para maestría y doctorado.

16. Como incide el emprendimiento en la satısfacción de los estudiantes de la Facultad de Ciencias de la Admınıstración - UNCP 2016

17. Calıdad de servicios y satısfacción de los estudiantes del pre grado del x ciclo de la Facultad de Ciencias de la Adminıstración.UNCP año 2016-II

18. La tutoría en la Facultad de Ciencias de la Adminıstración: análısıs, evaluación y propuesta.
En la Tabla 2, se muestra el ámbito de las 18 investigaciones expresadas en porcentaje; de los cuales 11 , son realizadas al interno de la FCA; 2 realizadas en la UNCP; 3 para la elaboración de libros y; 2 realizadas fuera de la UNCP.

Tabla 2

Clasificación porcentual de los trabajos realızados en FCA - UNCP

\begin{tabular}{lcc}
\hline \multicolumn{1}{c}{ Ámbito de las investigaciones } & $\mathbf{N}^{\circ}$ & $\mathbf{\%}$ \\
\hline $\begin{array}{l}\text { Investigaciones realizadas en la Facultad de } \\
\text { Ciencias de la Administración - UNCP }\end{array}$ & 11 & 61 \\
Investigaciones realizadas en la UNCP & 2 & 11 \\
Libros & 3 & 17 \\
Investigaciones realizadas fuera de la UNCP & 2 & 11 \\
\hline \multicolumn{1}{c}{ Totales } & $\mathbf{1 8}$ & $\mathbf{1 0 0}$ \\
\hline
\end{tabular}

La Tabla 3, expone las preguntas mas relevantes extraídas del cuestionario, demostrando las estrategias que utiliza la FCA en cuanto a la transferencia de tecnología y conocimiento, evidenciándose que; en la pregunta 1 , los 33 investigadores respondieron no; en la pregunta 2, 7 respondieron si y 28 no; en la pregunta 3, 4 investigadores respondieron si y 29 no; en la pregunta 4, 3 investigadores respondieron si y 30 no y; en la pregunta 5, 6 respondieron si y 27 no.

Tabla 3

Preguntas extraidas del cuestionario para determinar las estrategias utılızadas para la transferencia tecnológıca en la FCA

\begin{tabular}{llc}
\hline \multicolumn{1}{c}{ Preguntas } & Si & No \\
\hline $\begin{array}{l}\text { existe un personal especializado que guie en } \\
\text { la elaboración de proyecto }\end{array}$ & 0 & 33 \\
$\begin{array}{l}\text { El Instituto de Investigación, cuenta con con- } \\
\text { venios vigentes realizados por la Facultad }\end{array}$ & 7 & 28 \\
$\begin{array}{l}\text { Cuentas con un apoyo económico suficiente } \\
\text { para realizar una investigación }\end{array}$ & 4 & 29 \\
$\begin{array}{l}\text { La Facultad brinda la infraestructura adecua- } \\
\text { da para realizar la investigación }\end{array}$ & 3 & 30 \\
$\begin{array}{l}\text { Las investigaciones que realizas son multi- } \\
\text { disciplinarias }\end{array}$ & 6 & 27 \\
\hline
\end{tabular}

Se elaboró un cuestionario con 22 preguntas, con $\alpha$ de Cronbach $=0.848$. Se utilizó la prueba rho de Spearman con un resultado de 0.81 , porque se quiso demostrar si existe una relación entre las estrategias manejadas por el centro de investigación que viabilicen la transferencia de conocimiento y tecnología, el resultado evidencia una relación positiva fuerte.

\section{Discusión}

La forma de asegurar la transferencia de conocimiento y tecnología es aplicando el binomio universidad- empresa. En la FCA, se encontró que de 18 trabajos de 
investigación solo 2 (11\%) intentaron asegurar dicha transferencia. Esto viene a confirmar lo expuesto anteriormente; es decir, las empresas manufactureras invierten mayores recursos en adquirir tecnologías listas, en vez de invertir en proyectos de desarrollo tecnológico que los vincule con los centros de investigación locales. (Concytec, 2016). La universidad y la FCA deben de realizar convenios con empresas de la región Junín, en los cuales deben mencionarse las dificultades o problemas de la empresa a fin de solucionarlos, de esta manera los investigadores tendrán opción de elegir temas que aseguren una transferencia tecnológica.

A pesar que los 25 docentes nombrados realizan investigación, debería promoverse la formación de grupos multidisciplinarios, para asegurar la calidad de la investigación. En ese sentido, tienen que tener habilidades para relacionarse y entender a los empresarios y, al mismo tiempo, a los investigadores. Adicionalmente, es necesario crear una red de profesionales que permita el aprendizaje por medio de la difusión de las buenas prácticas en transferencia tecnológica y casos de éxito. (Concytec, 2016). Si las investigaciones son realizadas por grupos multidisciplinarios, el aporte sería enriquecedor, porque tendrían la óptica de diversas especialidades, alcanzando mejores soluciones.

Los trabajos se realizan dentro de la universidad o FCA, por la facilidad de la información y el bajo presupuesto con el que cuentan los investigadores, como se menciona a continuación, los subsidios que reciben, principalmente las universidades públicas, son insuficientes para el desarrollo de algunas actividades sustantivas y la vinculación es una de las vías propicias para captar esos recursos adicionales, que sirvan de apoyo a la investigación y para otras actividades académicas. (Vázquez, 2017).

Los investigadores se sentirían incentivados a realizar investigaciones serias y multidisciplinarias; al contarse, con presupuestos para esta labor, asegurando la transferencia tecnológica.

El Instituto Especializado de Investigación de la FCA, no tiene personal de apoyo, ni personal especializado en las labores de investigación, solo trabaja el director, quien tiene sus actividades académicas y administrativas, en este contexto, el personal es la parte central en el proceso de transferencia, porque su rol incluye una importante labor de vinculación entre investigadores y profesionales en propiedad intelectual. (Estrada, 2009). La transferencia de tecnología requiere de una alta especialización en los diferentes procesos que implican su adecuada gestión, haciéndose necesario contar con profesionales con capacidad de poder conducir en forma eficiente estos procesos. Se requiere expertos en identificación de tecnologías con el potencial de ser transferidas, gestión de propiedad intelectual, valorización y comercialización; así mismo, estos profesionales tienen que tener la capacidad de poder identificar oportunidades y necesidades de mercado y poder traducirlo al lenguaje académico (Concytec, 2016).

Mala distribución de personal de apoyo en la universidad, los cuales deberían reubicarse en los centros de investigación de las facultades, en cuanto a los especialistas, las autoridades deben asegurar el financiamiento para contratarlos.

\section{Conclusiones}

- En consecuencia, la universidad conjuntamente con los institutos de investigación, son los responsables para que exista un acercamiento entre empresa - universidad y Estado, para que los investigadores puedan realizar estudios que beneficien a la sociedad; asimismo, se debe exigir la conformación por grupos multidisciplinarios para obtener resultados efectivos de sus proyectos.

- La universidad debe asegurar el financiamiento para las labores investigativas, además la vinculación universidad - empresa debe generar los recursos que incentiven al investigador y, de esta forma, la empresa recobre la confianza de las investigaciones que se generan en la universidad.

- Para lograr la vinculación es necesario reconocer la importancia de la implementación de los institutos de investigación, con personal eficiente y especializado en identificación de tecnologías con el potencial de ser transferidas, gestión de propiedad intelectual, valorización y comercialización.

- En la vinculación universidad - empresa, debe existir la participación activa del Estado, proporcionando subsidios necesarios para tal fin.

\section{Referencias bibliográficas}

Arechavala, R. (2011). Las universidades y el desarrollo de la investigación científica y tecnológica en México: una agenda de investigación. Revista de Educación Superior, 40(158), 41-57. Retrieved from http:// resu.anuies. $\mathrm{mx} /$ archives/revistas/Revista158_ S2A2ES.pdf

Asistente, P. (2005). Estudios gerenciales un modelo integral para evaluar el impacto de la transferencia de conocimiento interorganizacional en el desempeño de la firma Augusto Rodríguez Orejuela, 37-50.

Azagra, J. (2003). La contribución de las universidades a la innovación: efectos del fomento de la interacción universidad-empresa y las patentes universitarias, 268.

Barragán O, A. (2010). Factores críticos para la gestión 
eficaz de proyectos de innovación en centros públicos de investigación y desarrollo, 144. Retrieved from https: / / www.ses.unam.mx/integrantes/uploadfile/jzubieta/Barragan2010_Tesis.pdf

Beraza Garmendia, J. M., \& Rodríguez Castellanos, A. (2010). Estructuras de intermediación para la transferencia de conocimiento universitario: Las oficinas de transferencia tecnológica. Propiedad Intelectual, IX(13), 152-176.

CONCYTEC. (2016). Programa Especial de Transferencia Tecnológica 2016-2021., 2021, 90. https: / / doi.org/10.1007/s10457-017-0147-9

Escuder, G.-P., García Vázquez, A., \& Piñeiro García, J. M. (2010). Incidencia de las políticas de recursos humanos en la transferencia de conocimiento y su efecto sobre la innovación incidence of human resources policies in the transfer of knowledge and its effect on innovation, 16, 1135-2523. Retrieved from http://www.redalyc.org/ pdf/2741/274120089007.pdf

Falc, Y., Hern, I. C., Gald, C., Gonz, M., Qu, I., Asistente, P., ... Km, O. (2013). Innovación tecnológica para la producción de biológicos en la Universidad Médica de Camagüey: ejemplo de relación universidad-, 13(2), 372-392.

Fernández, I., Castro, E., Conesa Cegarra, F., \& Gutiérrez Gracia, A. (2017). Las relaciones universidad-empresa: Revista Venezolana de Gestión Tecnológica, 21(2). Retrieved from http://digital.csic.es/ handle/10261/13382

Gómez-Vargas maricela.gomez@upb.edu.co, M., \& Alsina mgarciaals@uoc.edu, M. G. (2015).Factores influyentes de la gestión del conocimiento en el contexto de la investigación universitaria. (Spanish). Informacion, Cultura y Sociedad, 8327(33), 29-46. Retrieved from http: / / search. ebscohost.com $/ \operatorname{login}$.aspx ?direct $=$ true $\& \mathrm{db}={ }_{\mathrm{O}}$ $\mathrm{fm} \& \mathrm{AN}=111426915 \&$ lang $=$ es\&site $=$ ehost-live

Helios Feria, V. (2009). Propuesta de un modelo de transferencia de conocimiento científico -tecnológico para México, 412.

Hernández Sampieri, R., Férnandez Collado, C., \& Baptista Lucio, P. (2010). Metodología de la investigación. https: / / doi.org/ - ISBN 978-92-75-32913-9

Huanca-López, D. R. (2004). La investigación universitaria de países en desarrollo y la visión de los académicos sobre la relación universidad empresa: universidades públicas de la región occidental de Bolivia., 272. https://doi.org/10.4995/Thesis $/ 10251 / 8380$
Iglesias, P. P. (2010). Las Spin-Off Universitarias como mecanismo de transferencia de tecnología y su impacto económico en base a la actividad de I + D + i, (April), 1-426.

López, G. (2009). Instituto Politécnico Nacional. La inspección y evaluación no destructiva por el método de ultrasonido en materiales y componentes, para el mantenimiento, la seguridad y la sustentabilidad de la infraestructura industrial, 100. Retrieved from http://tesis.bnct.ipn.mx/ dspace/bitstream / 123456789/8307/1 / PROCESTRAT.pdf

Lucio, I. F. de, Martínez, E. C., Cegarra, F. C., \& Gracia, A. G. (2000). Las relaciones universidad-empresa: entre la transferencia de resultado. Espacios, 21(2). Retrieved from http: / www.revistaespacios.com/ a00v21n02/60002102.html

Martínez, A., Director, R., Manuel, F., \& Cabrera, S. (2014). Tesis doctoral. Gestión de la investigación en el campo de la información en Cuba: camino a su evaluación. Retrieved from http://tesis.repo.sld. cu/831/1/Ailín_Martínez_Rodríguez.pdf

Mauricio, J., \& Mogollón, G. (n.d.). Universidad -empresa, para la I + D generado por grupos de investigación de la Universidad de Pamplona.

Medellín Molina, E. R. (2015). Diagnóstico de las oficinas de transferencia de conocimiento (OTC) en México, 178.

Merchán Hernández, C. (2010). Las relaciones universidad - empresa en los sistemas regional de innovación: Análisis de la comunidad autónoma de Andalucía.

Morales Rubiano M. E., Sanabria Rangel, P. E., \& Caballetro Martinez, D. (2015). Características de la vinculación universidad - entorno en la Universidad Nacional de Colombia Tt. Revista Facultad de Ciencias Económicas: Investigación y Reflexión, 23(1), 189-208. Retrieved from http: / / www.scielo.org.co/scielo.php?script $=$ sci_arttext\&pid=S0121-68052015000100011\&lan$\mathrm{g}=\mathrm{pt} \% 0$ Ahttp://www.scielo.org.co/pdf/ rfce/v23n1/v23n1a11.pdf

Nathalie, L., \& Lobo, G. (2004). Del conocimiento en el contexto de la relación universidad - sector productivo. Educere, 8, 507-516.

Proceso, E. L., Tecnolog, D. E. T. D. E., Updce, C., Pol, M. E. N., Tecnol, D., Garc, F. F., \& Espinosa, O. (2014). Instıtuto Polıtécnıco Nacıonal Secretaria de Investigación y Posgrado. Centro de Investigaciones Económicas, Administrativas y Sociales. 
Salazar Pavez, A. A., Andrés, A., \& Salazar, P. (2000). Modelo de implantación de Gc y Ti para la generación de ventajas competitivas.

Sánchez, P. P. I. (2010). Facultad de Ciencias Económicas y Empresariales. Tesis doctoral Las Spin-Off universitarias como mecanismo de transferencia de tecnología y su impacto económico en base a la actividad de I + D + i.

Una, E. N., La, U. D. E., Caribe, C., Industrial, E. I., \& Autónoma, U. (2013). Estructura de un centro de transferencia tecnologica: innovacion Doctora en estadística industrial y optimización de procesos, docente Universidad Autónoma del Caribe, Ivonne Inés Volpe Barrios Laura Andrea Altamiranda Echeverri, $1-10$.

Universitario, M., \& Decisión, D. S. De. (2011). Universidad Rey Juan Carlos Resumen.

Vázquez, E. R. (2017). Transferencia del conocimiento y tecnología en universidades. Revista Iztapalapa, 83(38), 75-95. https://doi.org/10.28928/revistaiztapalapa/832017/atc3/vazquezgonzalezer

Vilardy Naggles, W. (2015). Gerencia de la gestión tecnológica en las universidades públicas. Escenarios, 11(1), 117. https://doi.org/10.15665/esc. v11i1.185

Zapata Cantú, L. E. (2004). Los determinantes de la generación y la transferencia del conocimiento en pequeñas y medianas empresas del sector de las tecnologías de la información de Barcelona, 260. Retrieved from http://www.tdx.cat/handle/ $10803 / 3955$ 